\title{
Crash Energy Management of Vehicle Front-end Structures Considering Multiple Conditions: Modelling and Solution Method
}

\section{Danqi Wang}

Changsha University of Science and Technology

Junyuan Zhang ( $\nabla$ junyuan_zhang@yeah.net)

Jilin University

Shihang Wang

Jilin University

\section{Research Article}

Keywords: Crash energy management, Front-end structure, Modelling, Multiple conditions, Vehicle

Posted Date: October 18th, 2021

DOI: https://doi.org/10.21203/rs.3.rs-980864/v1

License: (c) (i) This work is licensed under a Creative Commons Attribution 4.0 International License.

Read Full License 


\title{
Crash Energy Management of Vehicle Front-end Structures Considering Multiple Conditions: Modelling and Solution Method
}

(n)

\author{
Danqi Wang ${ }^{1}$, Junyuan Zhang ${ }^{2,}$, Shihang Wang ${ }^{2}$ \\ 1 College of Automotive and Mechanical Engineering, Changsha University of Science \& Technology, Changsha, China \\ 2 State Key Laboratory of Automobile Simulation and Control, Jilin University, Changchun, China \\ Correspondingauthor: junyuan@jlu.edu.cn; junyuan_zhang@yeah.net (Junyuan Zhang)
}

\begin{abstract}
For the vehicle frontal crash development, matching the stiffness of the front end structures reasonably, i.e., impact energy management, can effectively improve the safety of vehicle. A multi-condition analytical model for vehicle frontal crash is construct by three dimensional decomposition theory. In the analytical model, the spring is used to express the equivalent stiffness of the local energy absorption space at the front-end structure. Then based on the analytical model, the dynamic responses and evaluation indexes of the vehicle in MPDB and SOB conditions are derived with input of crash pulse decomposition scheme. Comparing the actual vehicle crash data and the finite element simulation results with the calculation results of the proposed solution method, the error is less than $15 \%$, which verifies validity of the modeling and the accuracy of the solution. Finally, based on the solution method in the MPDB and the SOB conditions, the sensitivities of crash pulse decomposition scheme to evaluation indexes are analyzed to obtain qualitative rules which guide crash energy management. This research reveals the energy absorption principle of front-end structure during the frontal impact process, and provides an effective tool to manage the vehicle crash energy considering multi-condition.
\end{abstract}

Keywords: Crash energy management; Front-end structure; Modelling; Multiple conditions; Vehicle

\section{Introduction}

The crash process of automobile includes three parts: barriers, vehicles, and occupants, and is a complex dynamics system. According to the annual statistical report of road traffic accidents from the Chinese government, frontal impact of automobile accidents account for the highest proportion among all accident forms ${ }^{[1,2]}$. In the frontal crash, the crash energy is absorbed by the front-end structures of vehicle ${ }^{[3]}$. With the development of crash safety regulations, today's vehicle front-end structural design needs to meet the requirements of multiple conditions such as FRB (frontal rigid barrier), MPDB (mobile progressive deformable barrier), and SOB (small overlap barrier) ${ }^{[4,5]}$. This greatly increases the difficulty of vehicle safety design. From the perspective of dynamics, revealing the crash energy dissipation mechanism and constructing a dynamic model of the front-end structures, which solve energy management in the early design stage, is great significance to improving the safety of vehicle ${ }^{[6]}$.

The ultimate goal of vehicle frontal crash safety research is to protect the safety of occupants. In the crash process, the initial kinetic energy of occupant is dissipated through two ways: the deformation of the vehicle structure and the action of the restraint system ${ }^{[7]}$. According to statistics about existing vehicles, the structure absorbs more than $60 \%$ of the kinetic energy of the occupants and is the most important way to absorb energy ${ }^{[8,9]}$. Crash energy management is to control the dissipation of energy by designing the rigidity and deformation of vehicle structure, so as to protect the safety of the occupants. In the vehicle frontal crash process, the energy dissipation is related to the topology of auto-body and the transmission path of the impact force ${ }^{[10-12]}$.

In the theory of impact mechanics, the crash energy absorbed by vehicle is approximately equal to the product of the impact force and the deformation of structures. Assuming that the vehicle mass is constant during the crash process, the impact force is approximately equal to the product of the acceleration and mass (constant). Define crash energy per unit mass as energy density. Then, the energy density absorbed by the vehicle during the impact is the product of acceleration and structural deformation ${ }^{[8,13]}$. When vehicle hits FRB, almost all of the crash energy is absorbed by the structural deformation. In this process, the vehicle acceleration is also called crash pulse. The sum of the deformations of all structures of vehicle in the longitudinal direction (the driving direction) is the maximum dynamic crushing. The maximum dynamic crushing is obtained by the quadratic integration of crash pulse ${ }^{[12,14]}$. That is to say, the crash energy is the integral of the crash pulse in the displacement domain. Therefore, from the perspective of mechanics, the frontal crash energy management of vehicle is actually the design and the decomposition problems of crash pulse $\mathrm{e}^{[8]}$. 
The design problem of crash pulse has been extensively studied through theories, simulations, and experiments ${ }^{[15-17]}$. At present, there are relatively mature methods and qualitative conclusions for guiding engineering design of crash pulse ${ }^{[18,19]}$. In terms of engineering conclusions, without considering the engine layout, the high-low-height crash pulse can effectively reduce occupant injuries; the double-step crash pulse is suitable for vehicles considering the engine layout, and the higher first step, the lower second step are the better for occupant safety ${ }^{[20]}$. In terms of theoretical method, our research group has established an automatic optimization method for crash pulse considering multiple impact conditions ${ }^{[21]}$. As a continuation of the paper on multi-condition optimization of crash pulse, this article focuses on the decomposition of crash pulse.

In this paper, the crash pulse in displacement domain as the total energy target during the impact process is decomposed into absorption energies of sub-structures based on the topology and the load path of vehicle. To achieve this, it is necessary to find the correlation between the target crash pulse and the structural performance. From 2006 to 2011, a magic cube approach is proposed to decompose the crash energy into sub-structure design goals from the three dimensions of time, space, and size, and design the load of the front longitudinal beams through the dynamic topology optimization method ${ }^{[22,23]}$. In 2011 , the energy-absorbing space of the front-end structure is divided into four layer in the vertical direction according to the transmission path of impact force, and used to derive the design goal of energy absorption by the simplified model ${ }^{[24]}$. In 2016, the vehicle longitudinal energy management method and the lateral energy management method are summarized to divide the front-end structure longitudinally, and then decompose the energies of each area according to the horizontal arrangement of the energy absorbing structure ${ }^{[7,25]}$. In 2018, the energy absorption space of front-end structures is vertically layered and longitudinally segmented by the deformation mode of sub-structures to obtain the decomposing energy as the design targets of anti-collision beam, the energy absorbing box, and the longitudinal beam ${ }^{[12,13,26]}$.

At present, it is mainly focused on the research of crash energy management methods of vehicle front-end structure based on engineering experience in the FRB impact condition ${ }^{[27-29]}$. Considering the impact force transmission characteristics of FRB condition, the front-end structure of the vehicle is divided into space, and the crash pulse is decomposed according to the sub-space as the energy absorption target ${ }^{[12]}$. In addition to FRB condition, the vehicle also needs to absorb energy through the deformation of the front-end structure in other frontal impact conditions, such as MPDB impact condition which mainly test crash compatibility and SOB impact condition which mainly test the safety of the passenger compartment ${ }^{[30-32]}$. Therefore, how to manage the energy absorption of front-end structures to meet the performance requirements and evaluation indicators of multiple frontal crash conditions is an urgent problem in the vehicle design industry, and it is also the research purpose of this paper.

Based on the frontal multi-condition crash pulse design method studied in the previous stage, this paper proposes a multi-condition crash energy management method, that is, the decomposition method of crash pulse. The main contributions of this paper include the following three aspects: 1) three dimensional analytical model of vehicle front-end structure is constructed to describe the energy absorption space, impact load path and structural stiffness; 2) based on the proposed analytical model, the dynamic responses and evaluation indexes of vehicle and barrier in the MPDB and SOB conditions are derived to realize the crash pulse decomposition considering multiple conditions; 3) based on the analytical model, the sensitivity of the crash pulse decomposition scheme to the evaluation indexes is analyzed to obtain the qualitative crash pulse energy management strategy.

The paper is organized as follows: Section 2 introduces a mechanical analytical model of vehicle front-end structure. In Section 3, we derive the solution of analytical model in MPDB and SOB conditions. An empirical case and a simulation results of existing vehicles that have been in running in China are applied to verify this constructed model and solution method in Section 3. Analysis and discussion are conducted in Section 4. Finally, the conclusions of this paper are drawn in Section 5.

\section{Three dimensional analytical model of vehicle front-end structure}

\subsection{Three dimensional decomposition of energy absorption space}

In this section, the energy absorption space during a frontal impact can be determined: as shown in Fig. 1, $B$ represents the width of the whole vehicle; $H$ represents the vertical distance from the intersection point of pillar $\mathrm{A}$ and the front finger beam to the chassis; $D$ represents the longitudinal space $L$ in the forward compartment of the vehicle minus the engine or motor occupancy $E$. The total energy absorption space can be decomposed in longitudinal, lateral and vertical dimensions as 
follows, considering the overlap rate of impact conditions, topological structure of the car body and the layout position of the engine and motor.

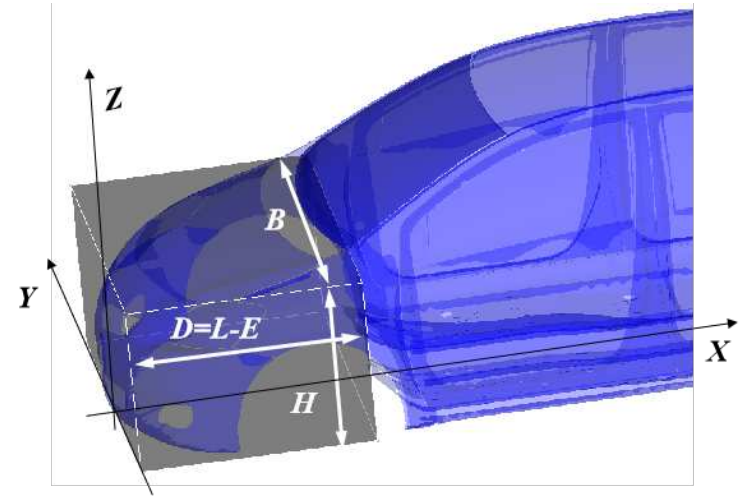

Fig. 1. The energy absorption space during a frontal impact.

Lateral decomposition: On both sides of the body symmetry plane, the energy absorption structure of the vehicle is almost exactly the same, and the contact area between the vehicle and the wall barrier in MPDB impact condition is also 50\% of the width of the vehicle. Thus, the total energy absorption space can be divided horizontally into two equal regions. To ensure a better safety level of the vehicle in the condition of $25 \%$ small bias impact, energy absorption structure should be set within $25 \%$ wide range on both sides of the vehicle. If the requirements of meeting various impact conditions are considered at the same time, four regions can be divided horizontally. The width of each region is $25 \%$ car width.

Longitudinal decomposition: According to the impact force transfer path of the vehicle in the frontal impact, the total energy absorption space can be vertically divided into two, three and four tiers longitudinally. Take a typical passenger vehicle as an example, which includes four tiers of the total energy-absorbing apace, the first tier is the engine cover, the second one is the front finger beam, the third one is the anti-impact beam, energy absorption box and longitudinal beam, and the fourth one is the sub-frame structure. The energy absorption is corresponding to the impact force. The energy absorption of each tier accounts for about $10 \%, 20 \%, 50 \%$ and $20 \%$ of the total energy absorption respectively ${ }^{[24]}$. The third tier includes the main energy absorption area of the vehicle. To improve the lightweight effect of vehicles, the engine hood is designed to be thinner and absorb less energy, so the energy absorption space is mainly divided into three tiers, that is, the first tier contains the engine hood, front finger beam and other structures. In addition, the sub-frame of some vehicles is removed in consideration of economic and lightweight factors, so that the longitudinal space is two tiers.

Vertical decomposition: The engine of traditional automobile and the motor of pure electric vehicle almost do not deform during the impact, which can be regarded as rigid structure. Therefore, according to the layout position of rigid components such as engine or motor, the total space of energy absorption can be divided longitudinally. For traditional gasoline cars and hybrid cars, rigid components such as engine need to be placed in the middle of the front end firewall considering the connection between engine and drive shaft and maintenance problems. Thus, the two sections from the front end of the engine to the anti-impact beam and from the back end of the engine to the firewall are set as impact energy absorption spaces. For pure electric vehicles, the motor can be considered close to the firewall layout, because there is no drive shaft on the vehicle. In this way, the whole from the anti-impact beam to the front end of the motor can be used as a section of energy absorption space.

In general, the three-dimensional decomposition of the total space of energy absorption at the front end of vehicle is to take into account the impact condition, the transmission path of impact force and the arrangement of the engine and motor, etc., and conduct horizontal division, vertical stratification and longitudinal segmentation successively. The decomposed fore cabin energy absorption space becomes the accumulation of energy absorption subspace. As a sample, the total space of energy absorption at the front end of vehicle is decomposed $4 \times 3 \times 2$ sections as shown in Fig. 2 . 


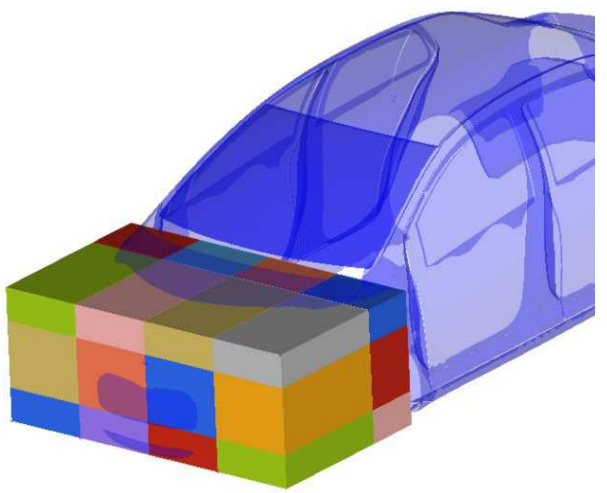

Fig. 2. Three-dimensional decomposition of the total energy absorption space of automobile front compartment.

\subsection{Three dimensional decomposition of crash pulse}

In the FRB impact condition, the wall is rigid, and the overlap rate between the vehicle and the wall is $100 \%$. This indicates that almost all the energy absorption structures in the front end of vehicle are involved in deformation energy absorption during the impact, and the acceleration response of the vehicle, namely the crash pulse, is also the result of the comprehensive action of the energy absorption structure. Thus, we take the crash pulse of FRB condition as the overall energy absorption objective of the vehicle's front end structure, and gradually decompose it into each energy absorption subspace and the design target of the energy absorption structure in space, so as to achieve the forward design of the front-end structure.

The horizontal coordinate of the crash pulse in the displacement domain is the deformation, which corresponds to the longitudinal direction of the energy absorption space, but there is no difference between the lateral direction and the vertical direction. Therefore, the three dimensional decomposition method proposed in this section is mainly to decompose the crash pulse into each energy-absorbing subspace in accordance with a given proportion according to the three-dimensional decomposition scheme of the total energy-absorbing space. If the longitudinal part of the total energy absorbing space is divided into two parts, the crash pulse is divided into two parts at the deformation corresponding to the longitudinal subspace.

Assuming that the front energy absorption space of the vehicle is divided into $N$ tiers vertically and $M$ zones horizontally, the decomposition scheme $Q$ of the crash pulse can be obtained as shown in Fig. 3. Note that the percentage of the absorbed energy of the sub-absorbent space in the total absorbed energy is represented by $q_{n m}(n=1,2, \ldots, N ; m=1,2, \ldots, M)$.

\begin{tabular}{|c|c|c|c|c}
\hline$q_{11}$ & $q_{12}$ & $\cdots$ & $q_{1 m}$ & $Q_{Z 1}$ \\
$q_{21}$ & $q_{22}$ & $\cdots$ & $q_{2 m}$ & $Q_{Z 2}$ \\
\hdashline$\vdots$ & $\vdots$ & $\ddots$ & $\vdots$ & $\vdots$ \\
$q_{n 1}$ & $q_{n 2}$ & $\cdots$ & $q_{n m}$ & $Q_{Z n}$ \\
\cline { 1 - 3 } & $Q_{Y 1}$ & $Q_{Y 2}$ & $\cdots$ & \multicolumn{2}{l}{$Q_{Y m}$}
\end{tabular}

Fig. 3. Preliminary decomposition scheme of frontal impact energy.

$$
\begin{aligned}
& Q=\left[\begin{array}{cccc}
q_{11} & q_{12} & \cdots & q_{1 M} \\
q_{21} & q_{22} & \cdots & q_{2 M} \\
\vdots & \vdots & \ddots & \vdots \\
q_{N 1} & q_{N 2} & \cdots & q_{N M}
\end{array}\right] \\
& \begin{cases}Q_{Y m}=q_{1 m}+q_{2 m}+\ldots .+q_{N m}, & m=1,2, \ldots, M \\
Q_{n Z}=q_{n 1}+q_{n 2}+\ldots .+q_{n M}, & n=1,2, \ldots, N\end{cases}
\end{aligned}
$$


In Eqs. (1) and (2), $Q$ represents the total absorbed energy; $Q_{Y m}$ represents the absorbed energy of $m$ th region, $m=1,2, \ldots$, $M ; Q_{Y m}$ represents the absorbed energy of $n$th tier, $n=1,2, \ldots, N$.

\subsection{Construction of analytical model}

After the three-dimensional decomposition of the total energy absorption space, each subspace corresponds to a decomposed crash pulse, that is sub-pulse. It is the same as the original pulse in shape, but the amplitude is different, which is the product of the original pulse and the proportion of energy absorbed by each subspace. The crash pulse in the displacement domain can be regarded as the equivalent specific stiffness of the front-end structure of the vehicle; similarly, the sub-pulse decomposed to each energy-absorbing subspace can be regarded as the equivalent specific stiffness of the space.

Fig. 4 represents the three-dimensional vehicle analytical model for frontal impact. In this model, the equivalent specific stiffness of each energy-absorbing subspace is expressed by spring stiffness $k_{i j}$, rigid bodies such as engine, motor and passenger cabin are expressed by a mass block.

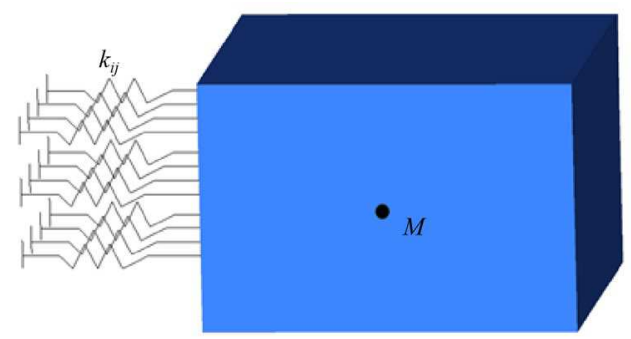

Fig. 4. Three-dimensional vehicle analytical model.

The spring stiffness $k_{i j}$ can be calculated as follows:

$$
k_{i j}=q_{i j} \times \frac{a_{v}}{d_{v}} \times M
$$

where, $a_{v}$ represents crash pulse; $d_{v}$ represents vehicle displacement; $M$ represents vehicle mass; $i$ is the number of lateral decomposition of the total space of energy absorption in the front compartment, and $j$ is the number of vertical decomposition of the total space of energy absorption.

\section{Solution of analytical model in multi-condition of vehicle frontal crash}

\subsection{Solution method for MPDB condition}

In the MPDB impact system, it mainly consists of two parts: barrier and vehicle. The impact process of MPDB condition is defined as the process in which the vehicle and the barrier start to contact either of them with a deceleration of 0 . It is assumed that the masses of the MPDB and vehicle are constant and the energy-absorbing structures of the two are only plastically deformed without elastic deformation during the impact process. Due to the rotations of vehicle and barrier are all small during the impact process, structural deformation is the most important way to absorb energy ${ }^{[21]}$. The simplified model of MPDB condition can be constructed ignoring the energy converted to rotation as shown in Fig. 5.

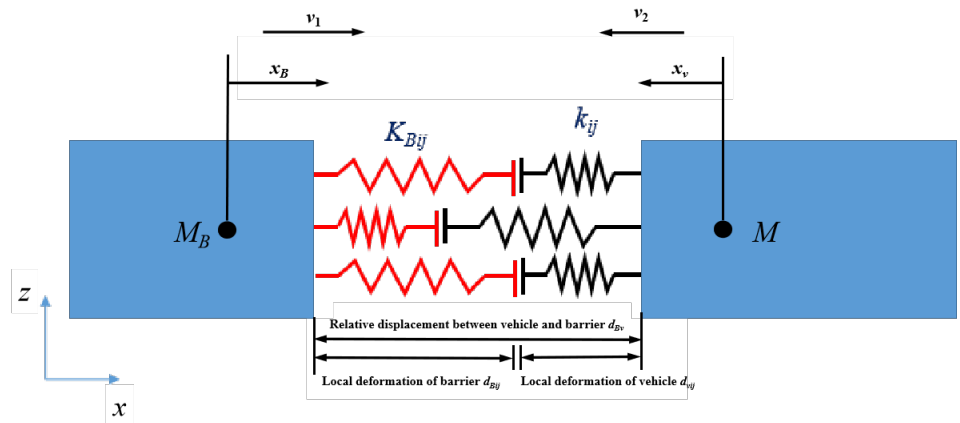

Fig. 5. The simplified model of MPDB condition. 
In this model, the barrier parameters obtained in the conceptual design stage include the mass and equivalent stiffness of the barrier, the general layout parameters of the vehicle, including the energy absorption space of the front-end structure and the mass of the vehicle as the system parameters. Take the crash pulse of vehicle FRB condition as input; The dynamic response of the vehicle and the barrier during the impact was solved, and the vehicle compatibility evaluation indexes are calculated.

In the Fig.5, the vehicle and the barrier move in relative motion; $M$ and $M_{B}$ are the masses of the vehicle and the barrier; $d_{B v}$ is their relative displacement; $d_{i j}$ is the local deformation of the vehicle; $d_{B i j}$ is the local deformation of the barrier; $k_{i j}$ is the local stiffness of the vehicle, and $k_{B i j}$ is the local stiffness of the barrier.

During the impact, the local stiffness of the vehicle and the barrier, $k_{i j}$ and $k_{B i j}$, are first connected in series to obtain the equivalent stiffness $k_{\text {eqij }}$ of each energy absorption subspace, and then the equivalent stiffness of each energy absorption subspace is connected in parallel to obtain the equivalent stiffness $K_{e q}$ of the vehicle and the barrier, as follows:

$$
\begin{aligned}
& \left\{\begin{array}{l}
k_{e q i j}=\frac{k_{B i j} \bullet k_{i j}}{k_{B i j}+k_{i j}} \\
K_{e q}=\sum k_{e q i j}
\end{array}\right. \\
& \left\{\begin{array}{l}
M_{e q}=\frac{M_{B} \cdot M}{M_{B}+M} \\
v_{e q}=\frac{v_{0} \cdot v_{0}}{v_{0}+v_{0}}=\frac{v_{0}}{2}
\end{array}\right.
\end{aligned}
$$

where, $M_{e q}$ represents the equivalent mass of the model, and $v_{e q}$ represents the equivalent velocity.

The motion response of the vehicle and the barrier can be obtained as follows:

$$
\left\{\begin{array}{l}
d_{B}(t)=\frac{M}{M_{B}+M} \cdot \frac{2 v_{0}}{\omega} \sin (\omega t) \\
d_{v}(t)=\frac{M_{B}}{M_{B}+M} \cdot \frac{2 v_{0}}{\omega} \sin (\omega t)
\end{array}\right.
$$

$$
\left\{\begin{array}{l}
v_{B}(t)=v_{0}-\int a_{B}(t) d t \\
v_{v}(t)=v_{0}-\int a(t) d t
\end{array}\right.
$$

$$
\omega=\sqrt{\frac{K_{e q}}{M_{e q}}}
$$

where, $d_{B}$ and $d_{v}$ are respectively for the barrier and the deformation of the vehicle impact process; $a_{B}$ and $a_{v}$ are respectively for the barrier and the vehicle acceleration, $v_{B}$ and $v_{v}$ are the barrier and the vehicle speed, respectively; $\omega$ is the natural frequency of the impact system.

In addition, three compatibility evaluation indexes of MPDB condition, i.e., the relative motion displacement of the vehicle and the barrier $\left(d_{B v}\right)$, the uniformity index of the barrier $(S D)$ and the maximum deformation of the barrier $(M D)$, as follows:

$$
\begin{gathered}
d_{B v}=d_{B}+d_{v} \\
d_{B i j}=d_{B v} \times \frac{k_{i j}}{k_{B i j}+k_{i j}} \\
S D=\sqrt{\frac{1}{m} \times \sum\left(d_{B i j}-\bar{d}_{B i j}\right)^{2}} \\
d_{B \max }=\max \left(d_{B i j}\right)
\end{gathered}
$$

where, $m$ represents the number of forward compartment subspaces that absorb energy 


\subsection{Solution for small overlap condition}

In the small overlap condition, the vehicle decelerates along the longitudinal direction and rotates around its contact point with the rigid barrier after contacting with the barrier. If one of the following two point occurs, the impact process of small overlap condition is considered to be over: 1 ) when the speed of the vehicle decreases to 0 , the structures of vehicle no longer deforms longitudinally; 2 ) if the vehicle displacement in the $\mathrm{Y}$ direction is $\geq 25 \%$ of the vehicle width, the vehicle is detached with the barrier with out structural deformation. Therefore, the vehicle motion responses in the impact process of small overlap condition can be considered from two aspects, i.e. the deceleration motion and rotation motion of the vehicle.

Based on the theory of impact mechanics, the simplified model of small overlap condition can be obtained as shown in Fig. 6. In this model, $O$ is the contact point between the vehicle and the barrier, and also the rotation center of the vehicle. $v_{0}$ is the impact speed, $M$ is the mass of the vehicle, and $k_{i j}$ is the vehicle stiffness.

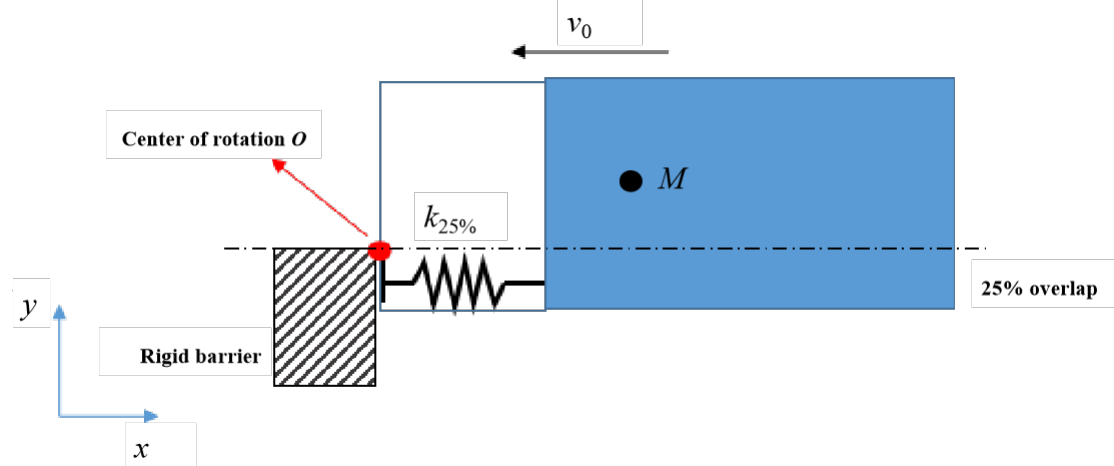

Fig. 6. The simplified model of small overlap condition.

The motion response of the vehicle in this condition can be considered from two aspects, i.e., the deceleration motion and the rotation motion of the vehicle. The $25 \%$ overlap area between the vehicle and the barrier is mainly the energy-absorbing area during the impact, its equivalent stiffness $\left(k_{25}\right)$ can be calculated as follows:

$$
k_{25 \%}=\sum k_{i 2}
$$

The vehicle makes a single-degree-of-freedom free vibration in the $\mathrm{X}$-axis direction. The vibration equation is as follows:

$$
M \ddot{x}(t)+k_{25 \%} x(t)=0
$$

where, $x(t)$ is the displacement of the vehicle, and $\ddot{x}(t)$ is the acceleration response of the vehicle.

$$
\begin{gathered}
\omega=\sqrt{\frac{k_{25 \%}}{M}} \\
\left\{\begin{array}{l}
x(t)=A \sin (\omega t+\varphi) \\
\dot{x}(t)=A \omega \cos (\omega t+\varphi) \\
\ddot{x}(t)=-A \omega^{2} \sin (\omega t+\varphi)
\end{array}\right.
\end{gathered}
$$

In this condition, $t=0, x=0$ and $\dot{x}=v_{0}$. Thus, the motion response of the vehicle can be obtained as follows:

$$
\left\{\begin{array}{l}
x_{v}=x(t)=\frac{v_{0}}{\omega} \sin (\omega t) \\
v_{v}=\dot{x}(t)=v_{0} \cos (\omega t) \\
a_{v}=\ddot{x}(t)=-v_{0} \omega \sin (\omega t)
\end{array}\right.
$$

The force analysis of the vehicle under the condition of small overlap condition is shown in Fig. 7. The vehicle will rotate around point $O$ under the action of impact reaction $F$, and the distance between the vehicle's center of mass and the center of rotation is the radius of rotation $r$. When the vehicle is decelerating, its longitudinal displacement is $x_{v}$, and the longitudinal 
distance between the center of mass and the center of rotation is $1 / 2 L-x_{v}$. When the vehicle is rotating, the transverse displacement of the center of mass is $y$, and the transverse distance from the center of mass to the center of rotation is $25 \% B+y$.

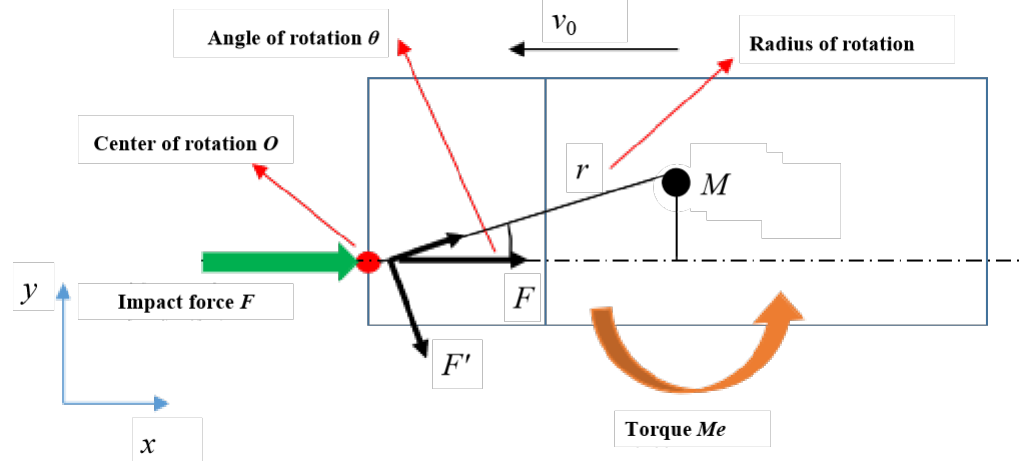

Fig. 7. The simplified model of small overlap condition.

The radius of rotation $(r)$ and the rotation angle $(\theta)$ of the vehicle can be calculated as follows:

$$
\begin{gathered}
r=\sqrt{(y+25 \% \times B)^{2}+\left(1 / 2 \times L-x_{v}\right)^{2}} \\
\theta=a \tan \left(\frac{y+25 \% \times B}{1 / 2 \times L-x_{v}}\right)
\end{gathered}
$$

The torque of the vehicle $(M e)$, the angular acceleration of the vehicle rotation $(\beta)$ and the lateral displacement of the vehicle $(y)$ can be obtained as follows:

$$
\begin{gathered}
M_{e}=F^{\prime} \cdot r=F \cdot \sin (\theta) \cdot r=F \cdot(y+25 \% B) \\
M_{e}=F^{\prime} \cdot r=J \cdot \beta \\
y=r \sin \theta-25 \% \times B
\end{gathered}
$$

where, $F^{\prime}$ represents the component force of the impact reaction $F$ perpendicular to the direction of the radius of rotation; $J$ represents the moment of inertia of the vehicle.

The maximum longitudinal displacement of the vehicle is the structural deformation of the vehicle in the process of impact. The intrusion of the crew compartment $\left(D_{25 \% \max }\right)$ can be calculated as follows:

$$
D_{25 \% \max }=\max \left(x_{v}\right)-L_{1}
$$

\subsection{Verification}

\subsubsection{Evaluation indexes}

1) Occupant load criterion $(O L C)$

The $O L C$ is calculated by the velocity-time curve of the barrier as Fig. 8 . The smaller $O L C$, the better ${ }^{[33]}$. At the time $t_{1}$ in the Fig. 8, the virtual occupant makes free movement relative to the barrier, and the displacement is $S_{1}=0.065 \mathrm{~m}$; from the time $t_{1}$ to $t_{2}$, the relative displacement between virtual occupant and barrier is $S_{2}=0.235 \mathrm{~m}$.

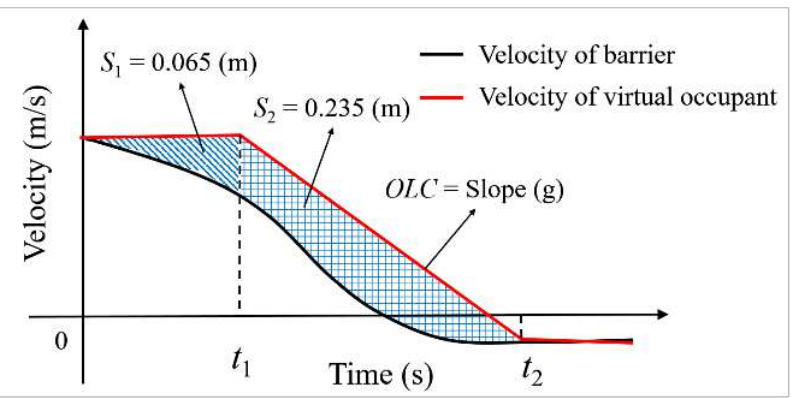

Fig. 8. The $O L C$ calculated by velocity-time curve of barrier. 
2) Maximum deformation $(M D)$

If there is an intrusion depth of $0.63 \mathrm{~m}$ in an area larger than $40 \mathrm{~mm} \times 40 \mathrm{~mm}$ on the barrier, the barrier is considered to be bottoming out ${ }^{[31]}$. At the time, deduction of two points is $M D=2$, otherwise $M D=0$.

3) Standard deviation $(S D)$

The $S D$ of barrier intrusion is obtained by the homogeneity of footprint based on scans of barrier ${ }^{[31]}$. The smaller $S D$, the better. The Barrier deformation uniformity factor $h$ is calculated by $S D$ as follows:

$$
\begin{cases}\text { if } S D<50 \mathrm{~mm}, & h=0 \\ \text { if } 50 \mathrm{~mm}<S D<150 \mathrm{~mm}, & h=(S D-50) / 100 \\ \text { if } S D>100 \mathrm{~mm}, & h=1\end{cases}
$$

4) The penalty of Mcompat (PM)

The calculation rules for $P M$ are shown in the following ${ }^{[31]}$ :

$$
\left\{\begin{aligned}
& \text { 1. } O L C<25 g: \\
& \text { Mcompat }=-2 \times h-M B O ; \\
& 2 . \quad 25 g \leq O L C \leq 40 g: \\
& M \text { compat }=-2 \times O L C / 15+10 / 3 \\
&-h \times((4 \times O L C / 10-8) \\
&-(2 \times O L C / 15-10 / 3))-M B O ;
\end{aligned}\right.
$$

3. $O L C>40 g$ :

$M$ compat $=-2-6 \times h-M B O$.

\subsubsection{Verification of MPDB condition}

The data about three quality grade vehicles in FRB and MPDB tests ${ }^{[30]}$, are used to verify the accuracy and reliability of solution method. In this condition, to research the influence of pulse parameters and vehicle quality on the evaluation index, including $O L C, M D, S D$ and $P M$, three kinds of vehicles, i.e., V1, V2, V3, with the masses of $1700 \mathrm{~kg}, 1400 \mathrm{~kg}, 1100 \mathrm{~kg}$, respectively, are applied. The crash pulses of V1, V2, V3 are shown in Fig. 9.

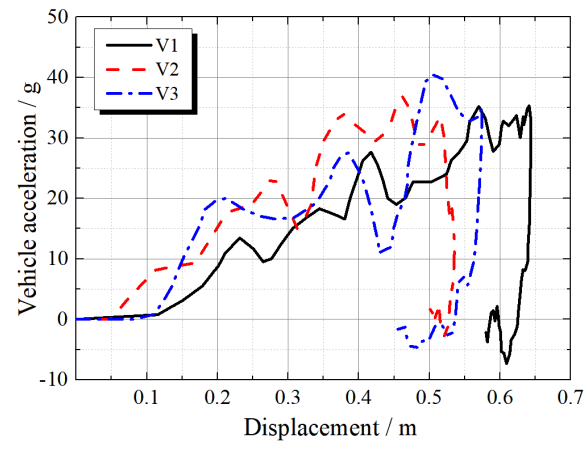

Fig. 9. The crash pulses of V1, V2, V3.

The stiffness decomposition scheme of the front structure of the three vehicles is all transverse $50 \%+25 \%$ zone and vertical three floors, denoted as " $4 \times 3$ " decomposition, as shown in Fig. 10. Decomposition plans $(Q)$ of V1, V2, V3 can be obtained as shown in Eq. 27.

\begin{tabular}{|c|c:c:c|l}
\hline$k_{12}$ & $k_{11}$ & $k_{11}$ & $k_{12}$ & $\boldsymbol{Q}_{z 1}$ \\
\hdashline$k_{22}$ & $k_{21}$ & $k_{21}$ & $k_{22}$ & $\boldsymbol{Q}_{z 2}$ \\
\hdashline$k_{32}$ & $k_{31}$ & $k_{31}$ & $k_{32}$ & $\boldsymbol{Q}_{z 3}$ \\
\cline { 1 - 3 } & $\boldsymbol{Q}_{\mathbf{Y} 1}$ & $\boldsymbol{Q}_{\mathbf{Y} 2}$ & $\boldsymbol{Q}_{\mathbf{Y} 3}$ & $\boldsymbol{Q}_{\mathbf{Y} 4}$
\end{tabular}

Fig. 10. The crash pulses of V1, V2, V3. 


$$
Q(\mathrm{~V} 1)=\left[\begin{array}{ll}
0.080 & 0.070 \\
0.120 & 0.080 \\
0.085 & 0.070
\end{array}\right], Q(\mathrm{~V} 2)=\left[\begin{array}{ll}
0.080 & 0.070 \\
0.110 & 0.090 \\
0.080 & 0.070
\end{array}\right], Q(\mathrm{~V} 3)=\left[\begin{array}{ll}
0.090 & 0.060 \\
0.100 & 0.100 \\
0.090 & 0.060
\end{array}\right]
$$

The values of $O L C, M D, S D$ and $P M$ can be obtained as Table 1. The errors between the calculation results and the existing data are all about $10 \%$, which indicate that the accuracy of the solution method is acceptable.

Table 1. The values of $O L C, M D, S D$ and $P M$

\begin{tabular}{cccccc}
\hline \multirow{2}{*}{ Vehicles } & Results & \multicolumn{4}{c}{ Evaluation indexes } \\
\cline { 3 - 5 } & & OLC $(\mathrm{g})$ & $M D(\mathrm{~m})$ & $S D(\mathrm{~mm})$ & $P M$ \\
\hline \multirow{2}{*}{ V1 } & Existing data & 35.9 & Bottoming, $M D>0.63$ & 56 & 3.74 \\
& Calculation & 31.30 & 0.71 & 63.34 & 3.38 \\
& Error & $-12.26 \%$ & Accord & $13.1 \%$ & $-9.6 \%$ \\
\hline \multirow{2}{*}{ V2 } & Existing data & 27.8 & NO, $M D<0.63$ & 93.5 & 1.57 \\
& Calculation & 27.33 & 0.62 & 98.3 & 1.58 \\
& Error & $-1.7 \%$ & Accord & $5.1 \%$ & $0.5 \%$ \\
\hline \multirow{2}{*}{ V3 } & Existing data & 26.3 & NO, $M D<0.63$ & 96.4 & 1.26 \\
& Calculation & 23.75 & 0.57 & 107.05 & 1.14 \\
& Error & $-9.7 \%$ & Accord & $11.04 \%$ & $-9.5 \%$ \\
\hline
\end{tabular}

\subsubsection{Verification of Small overlap condition}

The finite element calculation results of a vehicle in small overlap condition as the basic data is used to verify the accuracy of the analytical model by the maximum intrusion amount of the upper part of the passenger compartment. The vehicle mass is $1365 \mathrm{~kg}$, and the energy absorption space in the front compartment is $0.68 \mathrm{~m}$. The maximum intrusion value of the four intrusion measurement points on the upper part of the passenger compartment is taken as $34.2 \mathrm{~cm}$ in the simulation results of the vehicle as shown in Fig. $12^{[34]}$.

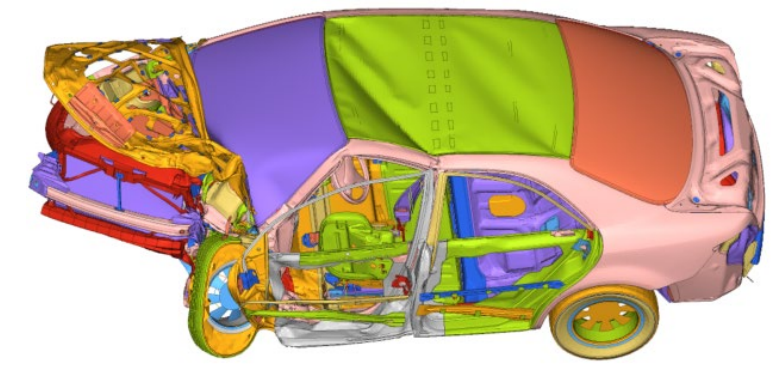

Fig. 11 The finite element calculation results of vehicle in FRB

It is known that the energy absorption decomposition scheme of the front-end structure of the car body is shown in Eq. 28. Take the crash pulse of the vehicle as shown in Fig.12 into the analytical model together with the decomposition scheme in Eq.28 to calculate the maximum longitudinal displacement of the vehicle as $1.0019 \mathrm{~m}$. The maximum intrusion value of the passenger compartment calculated by the analytical model is $1.0019-0.68=0.3219 \mathrm{~m}$, and the error with the maximum value of the upper intrusion of the passenger compartment of the finite element model is $-5.88 \%$. It shows that the analytical model solved in small overlap condition is effective.

$$
Q(\text { vehicle })=\left[\begin{array}{ll}
0.06 & 0.04 \\
0.21 & 0.10 \\
0.06 & 0.03
\end{array}\right]
$$




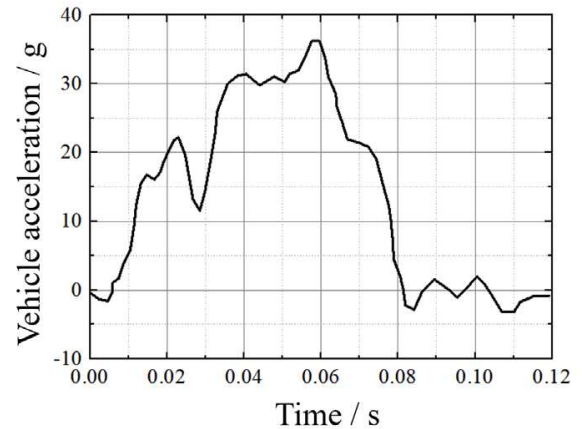

Fig.12 The crash pulse of vehicle in FRB condition

\section{Analysis and discussion}

\subsection{Impact analysis of different waveform decomposition schemes}

In this section, the effect of waveform decomposition scheme on three evaluation indexes of compatibility, i.e., $O L C$, $M D$ and $S D$, is studied. Note that the $4 \times 3$ decomposition method is adopted in this research. Considering the lateral symmetry of the car body, the variables of the decomposition method are the proportion of energy absorbed by six variables, and the sum of the six variables is $50 \%$, as shown in Eq. (28).

$$
Q=\left[\begin{array}{ll}
q_{11} & q_{12} \\
q_{21} & q_{22} \\
q_{31} & q_{32}
\end{array}\right], q_{11}+q_{12}+q_{21}+q_{22}+q_{31}+q_{32}=50 \%
$$

Three variation schemes for six variables are proposed, as follows:

Alternative 1: setting any one of the six variables to increase from 0 to $50 \%$, and the other variables are equal.

Alternative 2: setting any two of the six variables are increased from 0 to $50 \%$, and the other variables are equal.

Alternative 3: setting any three of the six variables and increase them from 0 to $50 \%$, and the other variables are equal.

The decomposition difference of each scheme can be calculated by Eq. (29). And the design and decomposition difference $(W)$ of the three alternatives are shown in Table 2.

$$
\begin{aligned}
W= & \left|q_{11}-q_{12}\right|+\left|q_{11}-q_{21}\right|+\left|q_{11}-q_{22}\right|+\left|q_{11}-q_{31}\right|+\left|q_{11}-q_{32}\right|+ \\
& \left|q_{12}-q_{21}\right|+\left|q_{12}-q_{22}\right|+\left|q_{12}-q_{31}\right|+\left|q_{12}-q_{32}\right|+ \\
& \left|q_{21}-q_{22}\right|+\left|q_{21}-q_{31}\right|+\left|q_{21}-q_{32}\right|+ \\
& \left|q_{22}-q_{31}\right|+\left|q_{22}-q_{32}\right|+ \\
& \left|q_{31}-q_{32}\right|
\end{aligned}
$$

Table 2. The design and decomposition difference of the three alternatives

\begin{tabular}{|c|c|c|c|c|c|c|c|c|c|}
\hline No & \multicolumn{3}{|c|}{ Alternative A } & \multicolumn{3}{c|}{ Alternative B } & \multicolumn{3}{c|}{ Alternative C } \\
\hline & One of $q_{i j}$ & Other $q_{i j}$ & $W$ & Two of $q_{i j}$ & Other $q_{i j}$ & $W$ & Three of $q_{i j}$ & Other $q_{i j}$ & $W$ \\
\hline 1 & 0 & 0.10 & 0.50 & 0 & 0.125 & 1.00 & 0 & 0.1667 & 1.5 \\
\hline 2 & 0.05 & 0.09 & 0.20 & 0.05 & 0.10 & 0.40 & 0.05 & 0.1177 & 0.6 \\
\hline 3 & $1 / 12$ & 0.0833 & 0 & $1 / 12$ & 0.0833 & 0.00 & $1 / 12$ & 0.0833 & 0 \\
\hline 4 & 0.1 & 0.08 & 0.10 & 0.1 & 0.075 & 0.20 & 0.1 & 0.0667 & 0.3 \\
\hline 5 & 0.15 & 0.07 & 0.40 & 0.15 & 0.05 & 0.80 & 0.15 & 0.0167 & 1.2 \\
\hline 6 & 0.2 & 0.06 & 0.70 & 0.2 & 0.025 & 1.40 & & & \\
\hline 7 & 0.25 & 0.05 & 1 & 0.25 & 0 & 2.00 & & & \\
\hline 8 & 0.3 & 0.04 & 1.30 & & & & & \\
\hline
\end{tabular}




\begin{tabular}{|c|c|c|c|l|l|l|l|l|l|}
\hline 9 & 0.35 & 0.03 & 1.60 & & & & & & \\
\hline 10 & 0.4 & 0.02 & 1.90 & & & & & & \\
\hline 11 & 0.45 & 0.01 & 2.20 & & & & & & \\
\hline 12 & 0.5 & 0 & 2.50 & & & & & & \\
\hline
\end{tabular}

In this section, the decomposition difference and the stiffness change position of the three schemes are adopted as the criteria to measure the uniformity of the barrier. The greater $W$ is, the poorer the uniformity of the decomposition scheme is. At the same $W$, the more varied the locations, the better the uniformity of the decomposition scheme. With $W$ as the horizontal coordinate, the values of $O L C, M D$ and $S D$ calculated by the three schemes are drawn respectively, as shown in Figs. 13.

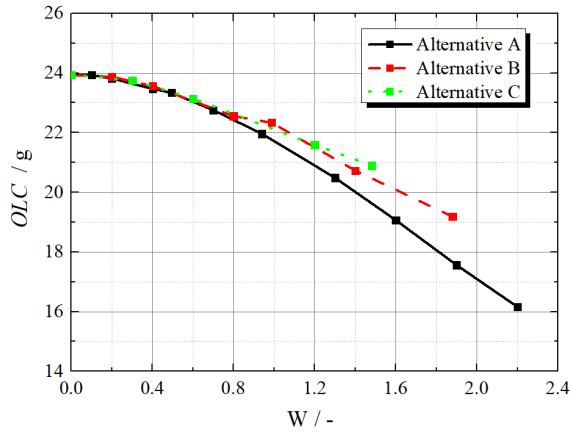

(a) $W$ VS. $O L C$

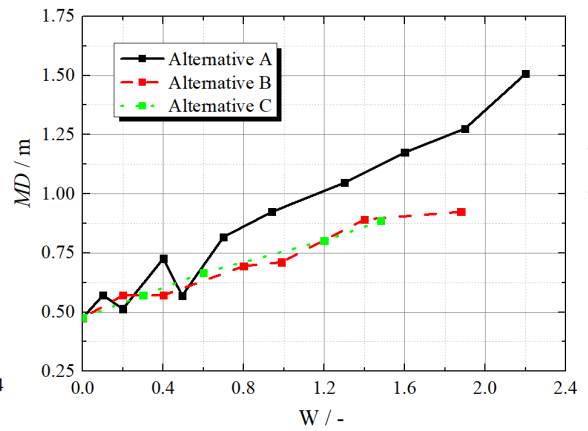

(b) $W$ VS. $M D$

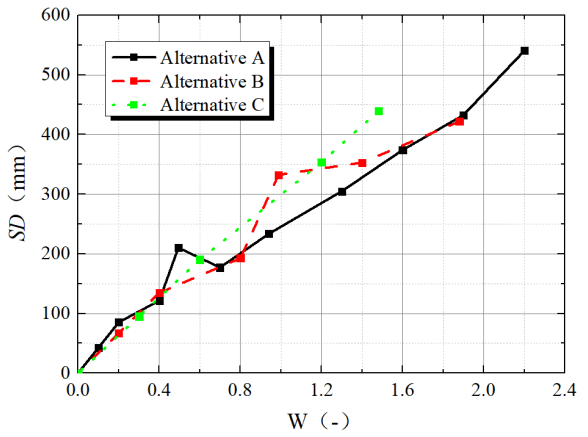

(c) $W$ VS. $S D$

Fig.13 The relationships between $\mathrm{W}$ and three compatibility evaluation indexes

When $W$ is 0 , the local stiffnesses of vehicle are evenly distributed. As the $W$ increases, the uniformity becomes worse. Through the above analysis and the information in Fig. 13, it can be found that when $W$ is 0 , the $O L C$ is the largest, the $M D$ is the smallest, and the $S D$ equals 0 . In the Fig. 13, as the $W$ increases, the decomposition scheme becomes more and more uneven, the $O L C$ value gradually decreases, the $M D$ and $S D$ value gradually increases. Corresponding to the same value of $W$, the positional relationship of local stiffness changes in the three stiffness decomposition schemes is: alternative $3>$ alternative $2>$ alternative 1 .

When $W$ is the same, the $O L C$ values of three alternatives are basically the same, which shows that $O L C$ is more sensitive to $W$, but has nothing to do with the position of the stiffness distribution. When the $W$ is the same, the increase in the stiffness change position helps to reduce the $M D$, and increase $S D$. It shows that $M D$ and $S D$ are more sensitive to the change position of local stiffness and $W$. In general, the worse the uniformity of the vehicle stiffness distribution, the smaller the $O L C$, the larger the $M D$ and $S D$. In order to ensure that the barrier is not penetrated, and to control the deformation uniformity index $S D$, it is necessary to reasonably allocate the stiffness of front-end structure.

\subsection{Discussion about stiffness decomposition of vehicle}

In the reference [21], an analytical model integrating barrier, vehicle and occupant is established to obtain not only the system dynamic responses but also the two evaluation indexes in MPDB (OLC and $M D$ ), known as BVO model, as shown in Fig 14. The BVO model system refers to simplified models with the elements of masses and springs. It is assumed that vehicle and barrier have constant masses during the impact process. The spring stiffnesses are used to represent the deformation energy absorption process of the structures. Since the overlap between vehicle and barrier are ignored in the modeling, the rotation responses of the BVO system and the local deformation index of barrier $S D$ can't be calculated.

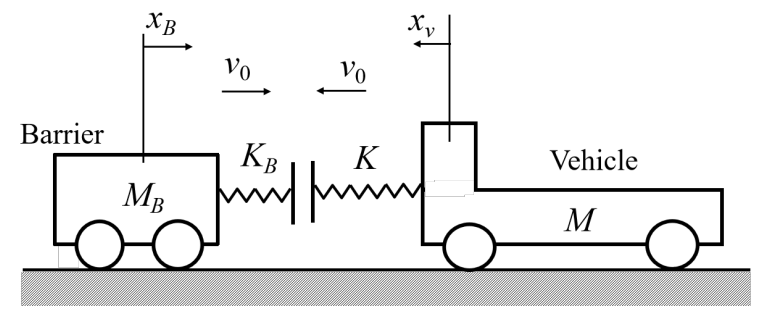


In order to solve the dynamic response of vehicle in the overlap impact conditions, the equivalent stiffness of the BVO model $\left(K_{B}\right.$ and $\left.K\right)$ are decomposed into local stiffness in this paper, as shown in Fig. 5. The three dimensional analytical model of vehicle front end structure after stiffness decomposition is shown in Fig. 4. The previous impact mechanics model can solve the one-dimensional dynamic response. The analytical model and solution method proposed in this paper provide new calculation ideas for the three-dimensional dynamic response of the impact system.

The vehicle data as the verification of the analytical model solved in MPDB test, are the same with data to verify the accuracy of BVO model in the reference [21]. The results of BVO model are calculated by the vehicle mass and crash pulse in the section 3.3.2, as shown in table 3 .

Table 3 The results of BVO model.

\begin{tabular}{lccc}
\hline & Large vehicle & Medium vehicle & Small vehicle \\
\hline$a_{v \max }(\mathrm{g})$ & 30.77 & 36.14 & 43.34 \\
$O L C(\mathrm{~g})$ & 33.15 & 29.17 & 25.84 \\
$M D(\mathrm{~m})$ & 0.63 & 0.55 & 0.48 \\
\hline
\end{tabular}

Comparing with the results calculated by the BVO model in Table 3, the $O L C$ values calculated by the analytical model are smaller and the $M D$ values are larger. This may be because that the local stiffnesses obtained after decomposing the vehicle stiffness are connected in parallel when the vehicle parameters are the same. The local stiffnesses of vehicle and barrier are first connected in series and then in parallel as the overall equivalent stiffness between the two to calculate the impact responses under MPDB condition. Therefore, if the decomposition is uniform, the equivalent stiffness between barrier and vehicle is constant, that is, the $O L C$ and accelerations of the system calculated by the BVO model and proposed analytical model are the same, and the deformation standard deviation $S D$ of the barrier is 0 . If it is not uniformly decomposed, the equivalent stiffness between vehicle and barrier is decreased, so that the $O L C$ is decreased; and a part of local stiffnesses of vehicle are increased to cause the local deformation of the barrier to increase, i.e. $M D$ is increased; At the same time, the $S D$ value is larger than 0 .

In engineering application, the main methods to improve vehicle compatibility are: reducing the quality of the entire vehicle, evenly distributing structural stiffness, and designing a reasonable crash pulse ${ }^{[12,13]}$. In the process of safety development, the quality level of the vehicle should be determined first, then the BVO model should be used to optimize the crash pulse, and finally the proposed model in this paper should be used to decompose the stiffness of front-end structure.

The effect of vehicle stiffness decomposition on the dynamic responses of small offset impact conditions is not analyzed in this paper. Theoretically, the greater the stiffness of the $25 \%$ area (QY1 or QY4 in the Fig.10), the smaller the deformation of the vehicle front-end structure and the smaller intrusion into the passenger compartment. Therefore, in the engineering design, the sum of the stiffness of the three positions in the $25 \%$ area should be increased as much as possible to improve the safety of the passenger compartment. That is to say, adding an energy-absorbing structures or increasing the stiffnesses of the existing structures in the $25 \%$ area can improve the safety of the vehicle in small offset impact condition effectively.

\section{Conclusion}

This paper studies the modeling method of vehicle front-end structures and the solution method of system dynamic responses in multiple impact conditions to manage the crash energy. In this paper, the energy absorption space of vehicle front-end is decomposed in three dimensions, and expressed by the spring stiffness to construct the analytical model of the front-end structure. Based on the three-dimensional analytical model of the front-end structure, the dynamic responses and evaluation indexes of the MPDB and SOB operating system are solved by the principle of engineering vibration with input of crash pulse decomposition scheme. Subsequently, the sensitivity of the crash pulse decomposition scheme to the evaluation indicators of each operating condition is analyzed based on the solution method in the multi-condition. Several concluding remarks are drawn as follows:

1) comparing the experimental data of the MPDB test and the calculation results of constructed analytical model, the errors of evaluation indexes, i.e., $O L C, S D$ and $P M$, are all less than $15 \%$, and judgments about the barrier bottoming out are all accord; 
2) comparing the simulation data of the SOB test and the calculation results of constructed analytical model, the errors of the maximum intrusion into passenger compartment is $-5.88 \%$;

3 ) as the $W$ increases, the decomposition scheme becomes more and more uneven, the $O L C$ value gradually decreases, the $M D$ and $S D$ value gradually increases;

4) $O L C$ is more sensitive to $W ; M D$ and $S D$ are more sensitive to the change position of local stiffness and $W$;

5) the greater the stiffness of the $25 \%$ area, the smaller the deformation of the vehicle front-end structure and the smaller intrusion into the passenger compartment.

In summary, this research reveals the energy absorption principle of front-end structure during the frontal impact process, and provides an effective tool to manage the vehicle crash energy considering multi-condition. In future research, our works will establish the theoretical relationship between the space energy absorption of the front-end structure and the energy absorption of the sub-structure to carry out the forward design of vehicle safety.

\section{Acknowledgements}

This work was supported by the National Natural Science Foundation of China (No. 51775228).

\section{Statements and Declarations}

Conflict of interest. The authors declare that they have no conflict of interest.

\section{Data availability statement}

The data used to support the findings of this study are available from the corresponding author upon request.

\section{Reference}

[1] Zhang H, Peng Y, Hou L, Tian G, Li Z (2019) A hybrid multi-objective optimization approach for energy-absorbing structures in train collisions. Information Sciences 481: 491-506.

[2] Zhang H, Peng Y, Hou L, Wang D, Tian G, Li Z (2020) Multistage impact energy distribution for whole vehicles in high-speed train collisions: modeling and solution methodology. IEEE Transactions on Industrial Informatics 16(4): 2486-2499.

[3] Duan L, Sun G, Cui J, Chen T, Cheng A, Li G (2016) Crashworthiness design of vehicle structure with tailor rolled blank. Struct Multidiscip Optim 53(2):321-38.

[4] Li QQ, Li E, Chen T, Wu L, Wang GQ, He ZC (2021) Improve the frontal crashworthiness of vehicle through the design of front rail. ThinWalled Struct 162:107588. https://doi.org/10.1016/j.tws.2021.

[5] Hu Lin, Bao Xingqian, Lin Miao, Yu Chao \&Wang Fang. Research on risky driving behavior evaluation model based on CIDAS real data, Proceedings of the Institution of Mechanical Engineers, Part D: Journal of Automobile Engineering, 2021, DOI:10.1177/0954407020985972

[6] Gidlewski, Mirosław, Prochowski L, Jemioł, Leszek, et al. The process of front-to-side collision of motor vehicles in terms of energy balance[J]. Nonlinear Dynamics, 2018.

[7] Qiu S (2016) Automobile Crash Safety Engineering, Institute of Technology Press. (In Chinese)

[8] Huang M (2002) Automobile crash mechanics, CRC press.

[9] Lin Hu, Xinting Hu, Aiwu Kuang, Miao Lin, Jie Wang. Casualty risk of e-bike rider struck by passenger vehicle using China In- depth accident data.Traffic Injury Prevention,2020, 21(4):283-287

[10] Reddy S R B . Multi-cornered thin-walled sheet metal members for enhanced crashworthiness and occupant protection[J]. Thin-Walled Structures, 2015, 94:56-66.

[11] Huang H, Siddiqui C, Abdel-Aty M (2011) Indexing crash worthiness and crash aggressivity by vehicle type. Accident Analysis \& Prevention 43(4): 1364-1370.

[12] Zhang J. (2018) parameters design method for structure and occupant restraint system in vehicle crash. Science Press. (In Chinese)

[13] Zhang J, Wang D, Wu L, Liu L (2019) Conceptual design of the front-end structure of automobile considering crashworthiness: a three-stage target decomposition method, International Journal of Crashworthiness, DOI:10.1080/13588265.2018.1550911

[14] K Mizuno (2016) Crash safety of passenger vehicles.[M] Beijing, China communications press.

[15] Zhang J, Wang D, Jin Y, Wu L (2018) The vehicle frontal impact safety evaluation and design method based on the coupling effect between crash pulse and restraint system characteristic. International journal of vehicle design 78(1-4): 79-107.

[16] Yang C, Li Q (2019) Further assessment of deceleration-time histories for occupant injury and the damage of protected object in a crash 
stop. International Journal of Impact Engineering 130:184-191.

[17] Xu P, Yang C, Peng Y, Yao S, Zhang D, Li B (2016) Crash performance and multi-objective optimization of a gradual energy-absorbing structure for subway vehicles. International Journal of Mechanical Sciences 107: 1-12.

[18] Shi Y, Wu J, Nusholtz GS. Optimal frontal vehicle crash pulses - a numerical method for design[C]/Proceedings of 18th Enhanced Safety of Vehicles (ESV) Conference, 2003, (514).

[19] Lars K., Simon G., and Konrad E (2009). Frontal crash pulse assessment with application to occupant safety. ATZ Worldw. 111 (6): $12-17$.

[20] Zhang J, Chen G, Liu L, Li H, Tang H (2012) Design and target decomposition of impact pulse of car frontal crashworthiness. Journal of Jilin University: Engineering and Technology Edition 42(4): 823-827. (In Chinese)

[21] Wang D, Zhang J, Ma Y, Jin Y. Analytical modeling and collaborative optimization of the dynamic responses for barrier-vehicle-occupant system considering crashworthiness and compatibility. Structural and Multidisciplinary Optimization, 2021, 64(1):349-367.

[22] Qi C, Ma ZD, Kikuchi N, et al. Fundamental studies on crashworthiness design with uncertainties in the system[C]// SAE Technical Paper, 2005.

[23] Qi C, Ma Z D, Kikuchi N, et al. Blast protection design of a military automobile system using a magic cube approach[C]// SAE Technical Paper, 2008.

[24] Malen DE, Fundamentals of automobile body structure design[M]. Warrendale Pennsylvania USA: SAE International, 2011.

[25] Zhou J, Qiu S, Zhang J, Ma Y (2015) Define occupant restraint system characteristics by using two-step trapezoid method based on ride-down rate control in frontal impact. International journal of vehicle safety 8(4): 314-338.

[26] Zhang J, Ma Y, Chen C, Zhang Y (2013) Design and Optimization for the Occupant Restraint System of Vehicle Based on a Single Freedom Model, Chinese Journal of Mechanical Engineering 26(3): 492-497.

[27] Mizuno K, Itakura T, Hirabayashi S, Tanaka E, Ito D (2014) Optimization of vehicle deceleration to reduce occupant injury risks in frontal impact, Traffic injury prevention 15(1):48-55.

[28] Teng T, Chang P, Liang C, Fung D (2017) Application of crash pulse on the car crashworthiness design. Advances in Mechanical Engineering 9(9):168781401770009.

[29] Yildiz A R, Solanki K N (2012) Multi-objective optimization of vehicle crashworthiness using a new particle swarm based approach. The International Journal of Advanced Manufacturing Technology 59(1-4): 367-376.

[30] Yan H, Zhong L, Yu J, Jiang W (2019) Research on 2020 E-NCAP MPDB Test. Automobile applied technology (21). (In Chinese).

[31] Euro N (2020) Roadmap European New Car Assessment Programme.

[32] Nguyen L P T . An optimisation approach to choose thickness of three members to improve IIHS small-overlap structural rating[J]. International Journal of Crashworthiness, 2017, 22(5-6):1-9.

[33] Lars K., Simon G., and Konrad E (2009). Frontal crash pulse assessment with application to occupant safety. ATZ Worldw. 111 (6): 12-17.

[34] Li L, Liu W, Zhang J, Zhang L, Zhou H. Design and improvement of a passenger vehicle frontal structure based on $25 \%$ small overlap frontal crash[J]. China Mechanical Engineering, 2015, 26(017): 2400-2405. 